\title{
Methodology based on collaborative problem solving implemented in a high academic achievement group
}

\section{Miguel Ferrando-Rocher, Stephan Marini, Juan José Galiana-Merino, Jesús Carbajo} Departamento de Física, Ingeniería de Sistemas y Teoría de la Señal, Universidad de Alicante, Spain.

\begin{abstract}
The High Academic Achievement (ARA, by its acronym in Spanish) group began its course in the 2011/12 academic year to reinforce the potential of the most outstanding students since the beginning of their university studies.

In order to improve the employability of this students, at least 50\% of basic or compulsory credits of the degree are taught in English. In addition, a series of language training aids are provided, which also has advantages in obtaining Erasmus scholarships. The ARA group only offers 25 places each academic course. Being a small group, personalized teaching is enhanced while the schedule is compacted.

In this contribution the methodology used in the subject of Signals and Systems of the Degree in Sound and Image in Telecommunication Engineering of the Alicante University (Spain) is presented. The methodology emphasizes the practical application of the subject and its direct applicability in real systems.
\end{abstract}

Keywords: Education; learning environments; partnership; collaboration; evaluation. 


\section{Introduction}

The ARA groups was promoted by the Valencian Ministry of Education in 2010. The ARA program is intended for university students who accredit better skills. The program provides all available aids and supports in order to obtain the highest possible academic performance. Currently, many universities in the Valencian public system have taken advantage of this teaching method.

The University of Valencia participates with the degrees in Medicine, Psychology, Law, International Business; with shared subjects in the degrees in Business Administration and Management, Tourism, Economics, Finance and Accounting;, and with subjects in the degrees in Pharmacy and Pharmacy and Human Nutrition. For its part, the Polytechnic University of Valencia has an ARA group in each of the following degrees: Biotechnology, Computer Engineering, Aerospace Engineering, Electronic, Industrial and Automatic Engineering, Business Administration and Management, and Technology and Services Engineering Telecommunications

At the the Universitat Jaume I, ARA groups are offerted in the shared subjects of the degrees in Industrial Technology Engineering, Chemical Engineering, Mechanical Engineering and Electrical Engineering.

For its part, the Miguel Hernández of Elche University has an ARA group in the Biotechnology Degree. Finally, the University of Alicante participates with ARA groups in the degrees in Computer Engineering, Law, Biology, and Sound and Image in Telecommunication Engineering. The present study focuses in this last engineering degree. Specifically, the ARA group of the subject of Signals and Systems, historically one of the subjects with the lowest passing rate among the second-year students.

Commonly, Signals and System subject of the Degree in Sound and Image in Telecommunication Engineering provides the student with the necessary tools on the basic concepts related to analog and digital signals and systems. These concepts are essential for future subjects in which signal processing is performed. The different types of analog and digital signals and systems are studied both in the time and frequency domainx, as well as the different tools that serve to characterize them in the corresponding transformed domains. The digitization of analog signals, which is necessary to perform digital signal processing, is also studied. The subject requires a non-negligible knowledge of the purely mathematical subjects studied in previous courses. However, this is the first time that students of this degree face a course where mathematics must be applied in real systems. Certainly, many times students do not have the required tools to understand the real application of the problems raised. 
Some of the objectives normally necessary to pass the subject include:

- Know and analyze the different elements and signals involved in telecommunication or remote communication, both analog and digital.

- Understand the process of digitizing analog signals.

- Develop the ability to analyze and design systems.

- To be able to characterize and analyze the signals and systems, continuous and discrete, in the time and frequency domains.

- Know the advantages and limitations of digital signal processing.

One of the potential challenges of this work has been to promote a flipped classroom system. Although the flipped classroom idea has been around for quite some time (Bland, 2006), it has recently been getting much interest for use in engineering courses at the university level. The basic premise of the flipped classroom is that active learning in the classroom is more effective than passive listening lectures. There is no doubt that active learning is effective in teaching signal processing (Fowler, 2014).

\section{Methodology}

The total students enrolled in the subject were 50, of which only 13 belonged to the high academic achievement group (as they must also fill in certain requirements).

Of the 13 students, 11 of them attended more than $90 \%$ of classes. Only some sporadic absences were recorded for justified reasons. The assistance was completely voluntary. Only two students attended less than $30 \%$ of classes.

The subject is structured in 5 major topics distributed during a period of 15 weeks, with 3 hours of class per week. The way to work each of the topics was to provide all the theory material the previous week of introducing a new topic. Students were encouraged to read it calmly.

In the first of the weekly sessions, the students were asked about all those doubts that had arisen in the theory. In this way, the teacher detected the general difficulties they have. Once the doubts were collected, there would be a debate for the whole class to explain these concepts. In this way, the most successful students in one part could strengthen their

knowledge and those who had not understood it could expose their doubts without fear in front of the rest of the classmates and the teacher. Obviously, this can be done since the number of students in the class is only 11. Hardly, if not impossible, such detailed monitoring could be done with more than 25 students. 
This theory review task used to last an hour. In case there were still doubts, which was common, the teacher encouraged students to come to tutorials so as not to slow down the progress of the class and advance the problems.

The following two hours per week established the following routine. By lot (weekly) workgroups were established, that way the groups were rotating and everyone helped everyone. Different problems were proposed with a degree of increasing difficulty that they had to solve in groups of 2 or 3 people. Finally, individually, they had to explain how they had reached the resolution of the problem in front of the rest of the class.

On the one hand, the way of working in group favored empathy with the partners and group work. On the other hand, the oral presentation challenged them to defend their knowledge in public so they knew that they should understand what they were doing (or if they do not understand, ask for an explanation).

This also favored the motivation for attendance and the challenge of wanting to solve the problems before the "competing" groups.

\section{Results}

In this section, the particular and preliminary results are presented. The way to evaluate the subject is more conventional since it is done through 3 partial individual and written exams, some problems proposed for each topic and a final exam. If the average of the three partials and the proposed problems is greater than 5, the final exam is not necessary.

On average, the final grade of the 11 students who actively attended class was 8.06 over 10 and none of them needed to attend the final exam. The two students who did not actively follow failed to pass either by continuous evaluation or with the final exam. When the students were consulted, their methodology was individual study. Fig. 1 summarizes the grades obtained by the 13 students in the group. It is noted that those who actively followed classes well approved all partials. Only low performance was observed in student 2 in the third part. All students attended more than $90 \%$ of the classes, except for students 1 and 6 , as previously mentioned. It is worth say that it must be carefully analyzed what has failed. For example, in the case of student 3 and what strategy can be used in those students who for whatever reason cannot actively follow the classes. 
FIRST PARTIAL EXAM

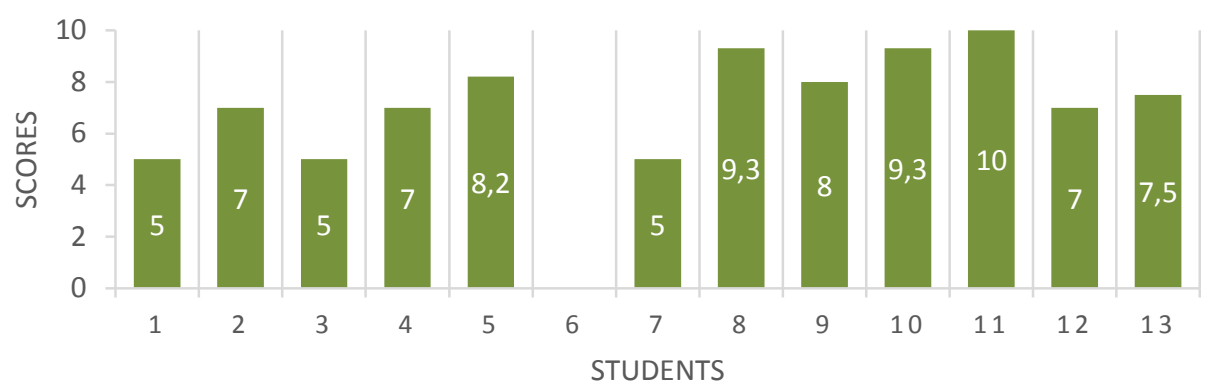

SECOND PARTIAL EXAM

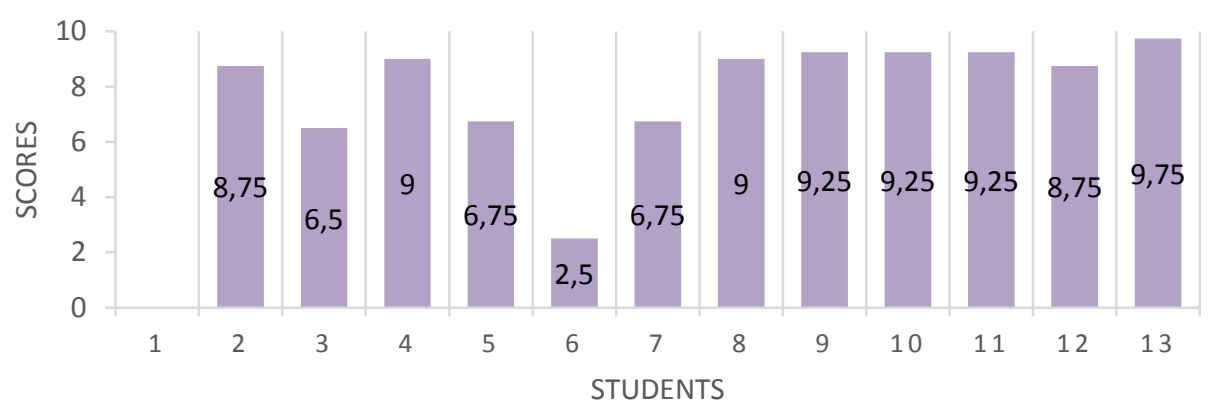

THIRD PARTIAL EXAM

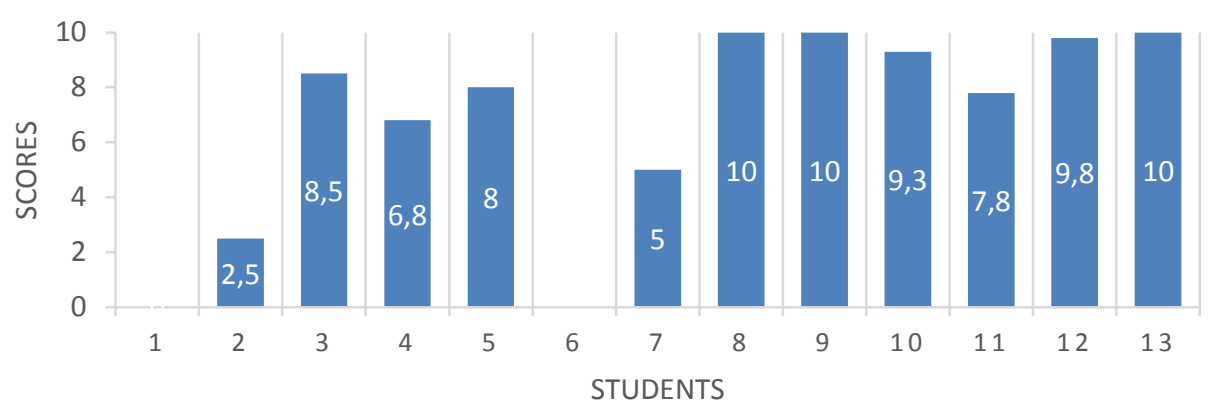

Figure 1. High-performance group ratings throughout the four-month period. 


\section{Conclusions and discussion}

It is usual for second-year students in technical careers to have difficulties when looking beyond a physical or mathematical problem and try to understand the applicability of the problem in a global way. There are many ways to approach and explain this connection between a problem and your future academic career. In this case, three fundamental tools have been chosen that make them think and not only have master classes where their participation and involvement is scarcer.

In the first place they must do an exercise of personal study, in the second a collective work and in the third place, a public and individual defense of the acquired knowledge. In addition, this methodology was constant throughout the four-month period, which gave them a routine, which, once achieved, is no different from any other.

The results validate that the methodology is very satisfactory, although certain considerations must be taken into account. In the first place it is a methodology suitable for small groups and secondly they are groups with students whose qualifications are over the average. In any case, the methodology has achieved that absolutely all the students who followed the methodology passed the subject. Even more important, all of them attended voluntarily and actively the classes from which it follows that they considered it a methodology useful for their studies and training.

\section{References}

Bland, L. (2006). Applying flip/inverted classroom model in electrical engineering to establish life-long learning. In ASEE Annual Conference \& Exposition (pp. AC2006856).

Fowler, M. L. (2014, May). Flipping signals and systems - Course structure \& results. In 2014 IEEE International Conference on Acoustics, Speech and Signal Processing (ICASSP) (pp. 2219-2223). IEEE. 